\title{
A "Multipurpose" 3D-Reconstruction Technique for Biplane Angiocardiography
}

\author{
Doriot P.-A., Dorsaz P.-A., Dorsaz L., Rutishauser W.
}

Cardiology Center, University Hospital, Geneva, Switzerland

\begin{abstract}
INTRODUCTION:
Despite the availability of highly sophisticated ultrasonic echocardiographs, computer tomographs (CT) and MRI equipments, angiocardiography remains indispensable for the precise diagnostic of the coronary artery disease, the most frequent cardiac pathology. Due to the spectacular development of "interventional cardiology" in the last 10 years, University hospitals are now usually equipped with "biplane" angiocardiographic units. These dedicated units are composed of two orientable gantries bearing each one an $\mathrm{x}$-ray tube and an image intensifier with attached TV and $35 \mathrm{~mm}$ cinefilm cameras. They allow to acquire nearly simultaneous images at high rates (typically 25 or 50 images per second in each two planes). Their main practical advantage is that the cardiologist can easily change the two projections or rapidly switch from one to the other one during intracoronary interventions (performed under fluoroscopic control). With regard to improved measurements of various parameters, the possibilities biplane units offer are, however, still under exploited. The purpose of this contribution is to describe some applications making use of the availability of images acquired nearly simultaneously in two incidences for 3D-reconstructions.
\end{abstract}

\section{D-RECONSTRUCTION TECHNIQUES AND APPLICATIONS:}

The first angiographic 3D-methods allowing for measurements of vessel lengths, arterial diameters or spatial angles were developed in the early sixties. Since in these methods the two perspective projections were approximated mathematically by two parallel projections, many distances and angles needed for 3D-calculations had to be determined and protocoled immediately before or after the acquisition of the two angiograms. In 1982, Mackay et al. proposed an improved technique in which the two perspective projections were described by two $4 \times 3$ matrices [1]. The two matrices were computed from two images of a cube acquired in exactly the biplane projection geometry used for the patient (The cube, of known side length, bears 15 steel markers: 8 at the corners, 6 centered on the faces and 1 at the center). Their method allowed for interactive 3D-reconstruction of one-dimensional structures like vessel centerlines. By help of an automatic detection of the vessel edges in the two angiographic images, the average vessel diameter value at each point of the spatial vessel centerline could also be determined. After completion of the $3 \mathrm{D}$-reconstruction, the vascular structure was pre- sented on the screen for inspection from different spatial view points. Further advantages of this technique are that it is relatively tolerant with regard to pincushion distortion, and that the two projection matrices contain implicitly the adequate calibration factors for every spatial location in the imaged 3D-zone.

Guggenheim et al. adapted then this technique to coronary arteries [2]. They showed that the lengths of coronary branches could be determined accurately (typically $+-2 \mathrm{~mm}$ for a $10 \mathrm{~cm}$ vessel segment), and that the accuracy of the computed intravascular volumes was somewhat less due to limitations in the determination of the local vessel diameters. This technique allowed to develop a method for assessment of mean flow in the left or right coronary artery based on a spatial approach instead of a temporal one, as used in earlier flow studies. In comparison with the techniques assuming two parallel projections, the recent 3D-techniques are not only more accurate [3] but also much less time consuming. Because of their possibilities for measurement of true vessel lengths and diameters, they can also be used to other purposes than flow measurements. In the following, we describe some other recent or potential applications.

A first example of 3D-measurements of arterial lengths and diameters is the study of Seiler et al. [4] of the relation between the total length of the measurable coronary vessels distally of an arbitrarily selected point of the coronary tree and the mass of myocardium supplied by these vessels. These authors examined also different theoretical models relating the cross sectional areas of the three vessel segments forming a bifurcation.

A second example is the improved assessment of coronary artery stenosis by densitometry. Contrary to morphometric methods based on diameter measurements, densitometry is not sensitive to the noncircularity of vessel lumens. It is therefore potentially superior to morphometric approaches. However, a practical problem is that the vessels of interest can often not be oriented adequately with res-pect to the $x$-rays of one or both planes, so that the obtained densitometric cross sectional areas are to great. 3D-reconstruction allows to determine the spatial angles between $x$-rays and vessel axis, and these angles can then be used to reduce the densitometric areas appropriately. It was shown that the discrepancies between the arterial cross sections obtained from the two planes in patients can be reduced by roughly a factor 2 [5]. In our-opinion, this improvement allows to consider 3D-densi- 
tometry as being now the best reference method for various comparisons. For instance, it can be used to compare the accuracy of stenosis assessment from a single projection to the accuracy achievable by use of two (more or less) orthogonal projections.

A third example involving 3D-reconstruction of coronary artery segments is the investigation of the impact of vessel movement during the cardiac cycle onto coronary flow pattern and wall shear stress [6].

A relatively simple and quick technique for approximate 3D-reconstructions has been developed by Solzbach et al. [7]. With their technique, a skeleton representation of the left or right coronary tree can be rapidly obtained during cardiac catheterization by pinning identifiable points of the vascular coronary configuration (e.g. vessel bifurcations) in a pair of biplane images. The approximate vessel centerlines are then found interactively by an iterative technique based on Bézier-curves. The interventional cardiologist can thus be quickly provided with a spatial representation of the coronary tree.

\section{OTHER POTENTIALLY INTERESTING APPLICATIONS:}

Many other potential applications seem also attractive. For instance, it is well known that stenoses occur often at vessel bifurcations. By measuring the spatial angles between the axes of the 3 vessels forming a bifurcation, one could investigate in patients whether coronary stenoses appear preferably at bifurcations where these angles have particularly great values. One could also investigate whether the occurrence of stenosis correlates with the amplitude of the cyclic evolution of these angles during the cardiac cycle. If such correlations would turn out to be appreciable, a new anatomical risk factor for coronary arteriosclerosis would have been identified.

Of practictal importance would be the evaluation of the mass of myocardium at risk of ischemia behind a stenosis. This problem could be approached by use of the relation between total length of the vessels distally of the stenosis and associated myocardial mass. Such an evaluation might provide a supplementary aid in deciding whether an intervention should be performed or not, or whether the patient should better undergo surgery.

A further but more problematic task could be the elaboration of a model allowing to predict the hemodynamical result of coronary artery surgery in function of the selected graft scheme. It can indeed occur that surgery does not quite result in the expected benefit due to a different hemodynamical redistribution of the arterial flows than anticipated. Such a method would, however, necessitate the measurement of many arterial lengths, cross sections and local intravascular pressures, and also a mathematical-physical model to combine all these values in a way appropriate to predict the hemodynamical consequences of the envisaged anastomosis site.
A 3D-representation of the left or right coronary tree could also be combined with a 3D-representation of the left ventricular chamber. The idea would be to design a model for 3D-visualization of left ventricular wall motion abnormalities computed from biplane left ventricular angiograms. The repercussion of a stenosis on the regional contraction could thus be better visualized than with the present models.

Interesting applications can also be envisaged in pediatric cardiology. For instance, 3D-reconstruction could provide the cardiologist during the cardiac catheterization procedure with a 3D-representation of the spatial configuration of the great cardiac vessels. In interventional neuro-radiology, 3Dreconstruction could be used to provide an estimation of the volumes of small cerebral aneurysms during the interventional procedure. These volumes are today computed from MRI views. To conclude this non exhaustive list of possible applications, let us finally mention the determination of the pulmonary volume set out of function by a pulmonary thrombus.

\section{CONCLUSION:}

Although CT and MRI offer potentially much better possibilities for $3 \mathrm{D}$-visualizations, the fact is that these two modalities are not (and may probably never be) suitable for coronary interventions. It is therefore important to develop new tools for improved quantitative assessments in the cardiac catheterization laboratory. The applications described in this contributions may prove valuable in this respect.

\section{REFERENCES}

[1] S.A. MacKay et al.; Comput. Biomed. Res. 15, 455(1982)

[2] N. Guggenheim et al.; Phys. Med. Biol. 36/1, 99(1991)

[3] G.T. Daughters et al.; Computers in Cardiology 1988, IEEE Computer Society, 79(1988)

[4] C. Seiler et al.; Circulation 85, 1987(1992)

[5] P.-A. Doriot et al.; Int. J. Cardiac Imaging (in press)

[6] J.E. Moore et al.; ASME Journal of Biomechanical Engineering 116, 302(1994)

[7] U. Solzbach et al.; Comput. Biomed. Res. 27, 178(1994) 